\title{
The ageing syndromes of sarcopenia and frailty
}

Charlotte Thompson

Richard M Dodds

Charlotte Thompson MB BS MRCPUK PGCE Medical Education is an ST3 in Geriatric Medicine and General Medicine at the Newcastle-upon-Tyne Hospitals NHS Foundation Trust, Newcastle, UK. Competing interests: None to declare.

Richard M Dodds PhD MRCP is a Clinical Fellow at Newcastle University and Honorary Consultant Geriatrician at Newcastle upon Tyne Hospitals NHS Foundation Trust, UK. Competing interests: None to declare

\begin{abstract}
Sarcopenia describes the accelerated loss of skeletal muscle, manifesting as a combination of low muscle strength and low muscle mass. Frailty can be described as the collective deterioration of multiple body systems, leading to reduced ability to maintain homeostasis and respond to physiological stresses placed upon the body. Frailty and sarcopenia are common, especially in hospitalized older people, and also occur at younger ages in the setting of multiple long-term conditions (multimorbidity). There are currently no drug treatments for sarcopenia and frailty, and this is one area of continuing research into these two syndromes. Their diagnosis in clinical practice helps to identify a group of patients at risk of adverse outcomes who could benefit from interventions including resistance exercise training and comprehensive geriatric assessment.
\end{abstract}

Keywords

Ageing; comprehensive geriatric assessment; frailty; grip strength; multimorbidity; resistance exercise; sarcopenia

Key points

- Sarcopenia and frailty are syndromes that become more common with age, and overlap in terms of the loss of skeletal muscle strength and mass

- They are linked to a range of adverse outcomes including increased risk of death; individuals with both frailty and sarcopenia tend to be at greater risk than those with sarcopenia alone

- The clinical assessment of sarcopenia and particularly frailty is becoming more widespread; once diagnosed, these syndromes should prompt a comprehensive geriatric assessment 


\section{Introduction}

Sarcopenia describes the accelerated loss of skeletal muscle, manifesting as a combination of low muscle strength and low muscle mass. Frailty can be described as a collective deterioration in multiple body systems, leading to reduced ability to maintain homeostasis and respond to physiological stresses placed upon the body. Sarcopenia and frailty are syndromes that become increasingly prevalent at older ages. They are important as both are associated with adverse healthcare outcomes including disability and mortality. Their assessment in clinical practice is becoming more widespread and provides an opportunity to identify individuals at risk of poor health and to begin relevant treatments. ${ }^{1}$

This review describes sarcopenia and frailty in terms of their diagnosis, epidemiology and management. The similarities and differences between these two ageing syndromes are highlighted throughout.

\section{Diagnosis}

Several diagnostic criteria for sarcopenia have been proposed, typically based on the combination of low muscle strength and low muscle mass. A recent example is the revised consensus definition of the European Working Group on Sarcopenia in Older People (EWGSOP). ${ }^{2}$ The first step is identifying possible cases of sarcopenia using the brief SARC-F questionnaire, which asks about five tasks including climbing a flight of stairs. Any difficulty with these tasks suggests a need for further assessment, as do a range of clinical risk factors including multimorbidity (the presence of two or more long-term conditions) and cognitive impairment.

The next step in the EWGSOP definition is to assess muscle strength, with two methods recommended for clinical practice. The first is grip strength, measured with a hand-held dynamometer (Figure 1), with three trials for each hand, the maximum value being used. Grip strength correlates with strength in other areas of the body, and low strength is indicated by a maximum grip strength of $<16 \mathrm{~kg}$ in women and $<27 \mathrm{~kg}$ in men. The second method is the chair stand test: the time taken to stand from seated five times as quickly as possible without using the arms. A time of 15 seconds or longer indicates low muscle strength. The next step to confirm sarcopenia in individuals with low strength is to assess muscle mass, specifically in the arms and legs (referred to as appendicular lean mass). A range of techniques is available: dual-energy X-ray absorptiometry scanning and bioelectrical impedance analysis are commonly used in clinical practice, and ultrasonography, computed tomography and magnetic resonance imaging used more in research.

Frailty can be diagnosed through three main approaches: the phenotypic model, the cumulative deficit model and the Clinical Frailty Scale (CFS). Fried et al. described a five-criterion phenotypic model of frailty consisting of weight loss, weakness, low physical activity, slow walking speed and exhaustion. If a patient has three or more of these criteria, they are considered to have frailty, with one or two criteria representing pre-frailty. Rockwood described the cumulative deficit model, based on the proportion of a number of possible deficits and symptoms present, including physical, sensory and cognitive impairments, and medical and psychiatric conditions. Rockwood also created the CFS, a pictorial representation of levels of functional impairment corresponding to a severity of frailty from 1 (very fit) to 8 (very severely frail). All three approaches to diagnosing frailty contain aspects of muscle strength or physical function, so there is a substantial degree of overlap between the definitions of sarcopenia and frailty.

\section{Epidemiology \\ Prevalence}

With the overlap in the features of sarcopenia and frailty, it follows that the prevalence of these syndromes follows similar patterns. Prevalence estimates vary with the exact definition used, but the prevalence of sarcopenia in one cohort with a mean age of 67 years was found to be $6.5 \%$, rising to $21 \%$ in another cohort aged 85 years. The prevalence of frailty using the phenotypic model was also found to be $6.5 \%$ at age $60-69$ years, rising to $31 \%$ in those aged $80-89$ years.

The presence of two or more long-term conditions (multimorbidity) is a risk factor for the syndromes of sarcopenia and frailty, and can lead to them being seen at younger ages. One study found a similar prevalence of probable sarcopenia in individuals aged 40-49 years with three or more categories of long-term conditions to those aged 60-70 years with only one (Figure 2). Individuals with frailty are highly likely to have multimorbidity: a previous meta-analysis found that $72 \%$ of individuals with frailty using the Fried definition had two or more long-term conditions. However, the converse was not seen: only $16 \%$ of individuals in the same study with multimorbidity were found to have frailty. 


\section{Associations with health outcomes}

Sarcopenia and frailty are associated with a range of negative health outcomes. These include the development or progression of disability, admission to long-term care, a greater risk of falls and reduced quality of life. The most striking outcome associated with sarcopenia and frailty is increased risk of death. Estimates for the strength of these associations vary with the definition used and the population studied. Sarcopenia appears to lead to a roughly doubling of risk of all-cause mortality. Frailty, as from the Fried definition, has been linked to at least this level of increased risk, often greater. ${ }^{3}$

\section{Management}

Patients with sarcopenia and frailty stand to benefit from treatments to improve muscle strength and mass. The strongest evidence is for the use of progressive resistance training. In frailer and less mobile individuals, the ability to take part in such exercise regimes can, however, be limited. There is less evidence that diet interventions are of benefit, although an increased protein intake of $1.0-1.2 \mathrm{~g}$ per kg body weight has been recommended in older adults. There is also evidence that replacement of vitamin $\mathrm{D}$ in women with low serum concentrations leads to improvements in strength and physical functioning. There are currently no licensed medications to treat sarcopenia and frailty, although research is continuing. ${ }^{4}$

The main focus of management for an older person with frailty is an individualized, holistic process known as comprehensive geriatric assessment (CGA). This is a multidisciplinary process, covering medical, functional, social and psychological elements of care, to provide a wide-ranging and personalized care plan aimed at promoting health and independence. Use of the CGA for individuals with frailty during an acute admission is associated with lower risks of both death and institutionalization. Further information on the management of frailty is provided in useful guidance from the British Geriatrics Society. ${ }^{5}$

\section{Relevance during acute hospital admission}

Sarcopenia is not commonly assessed during hospital admission, although there is growing awareness of the risks of deconditioning (including loss of muscle mass) during acute illness. In comparison, the assessment of frailty in older people, for example using the CFS, has become widespread on admission to hospital. The Acute Frailty Network

(www.acutefrailtynetwork.org.uk/about-us) is an improvement network that has helped hospitals to implement such tools. Importantly, the identification of frailty should lead to a prompt CGA; as described above, this has been shown to improve outcomes in frail older people admitted to hospital.

\section{Prevention}

There is interest in whether sarcopenia and frailty could be prevented, or delayed, by interventions earlier in life. As regards muscle strength, there is an increase in childhood and early adulthood, followed by broad maintenance in mid-life before loss in older age (Figure 3). It follows that factors influencing muscle strength in old age include those affecting the maximum level reached in early life and the rate of loss in older age. For example, evidence from a British birth cohort study suggests that increased leisure-time physical activity across adulthood is linked to stronger grip in old age, and hence a reduced likelihood of sarcopenia and frailty.

\section{Key References}

1. Dodds R, Sayer AA. Sarcopenia and frailty: new challenges for clinical practice. Clin Med 2015; 15(Suppl 6): 88-91.

2. Cruz-Jentoft AJ, Bahat G, Bauer J, et al. Sarcopenia: revised European consensus on definition and diagnosis. Age Ageing 2019; 48: 16-31.

3. Clegg A, Young J, lliffe S, Rikkert MO, Rockwood K. Frailty in elderly people. Lancet 2013; 381: $752-62$.

4. Cruz-Jentoft AJ, Sayer AA. Sarcopenia. Lancet 2019; 393: 2636-46.

5. British Geriatrics Society. Fit for frailty. Part 1. 2017.

https://www.bgs.org.uk/sites/default/files/content/resources/files/2018-05-23/fff_full.pdf (accessed 3 May 2020). 
Figures

Figure 1 Hand-held dynamometer to measure grip strength.

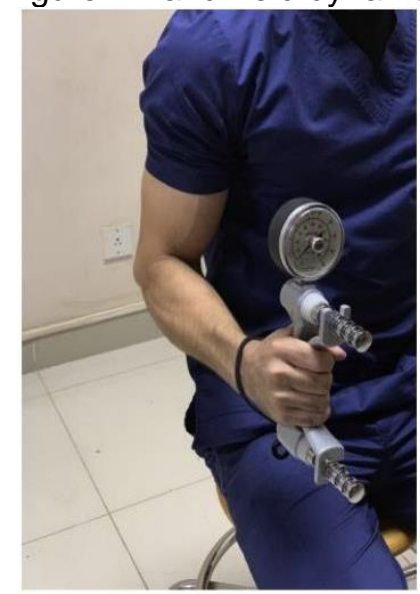

Source: Reproduced from El-gohary TM, Abd Elkader SM, Al-shenqiti AM, Ibrahim MI. Assessment of hand-grip and key-pinch strength at three arm positions among healthy college students: dominant versus non-dominant hand. J Taibah Univ Med Sci 2019; 14: 566-71. 
Figure 2 Probable sarcopenia by age and number of categories of long-term conditions.

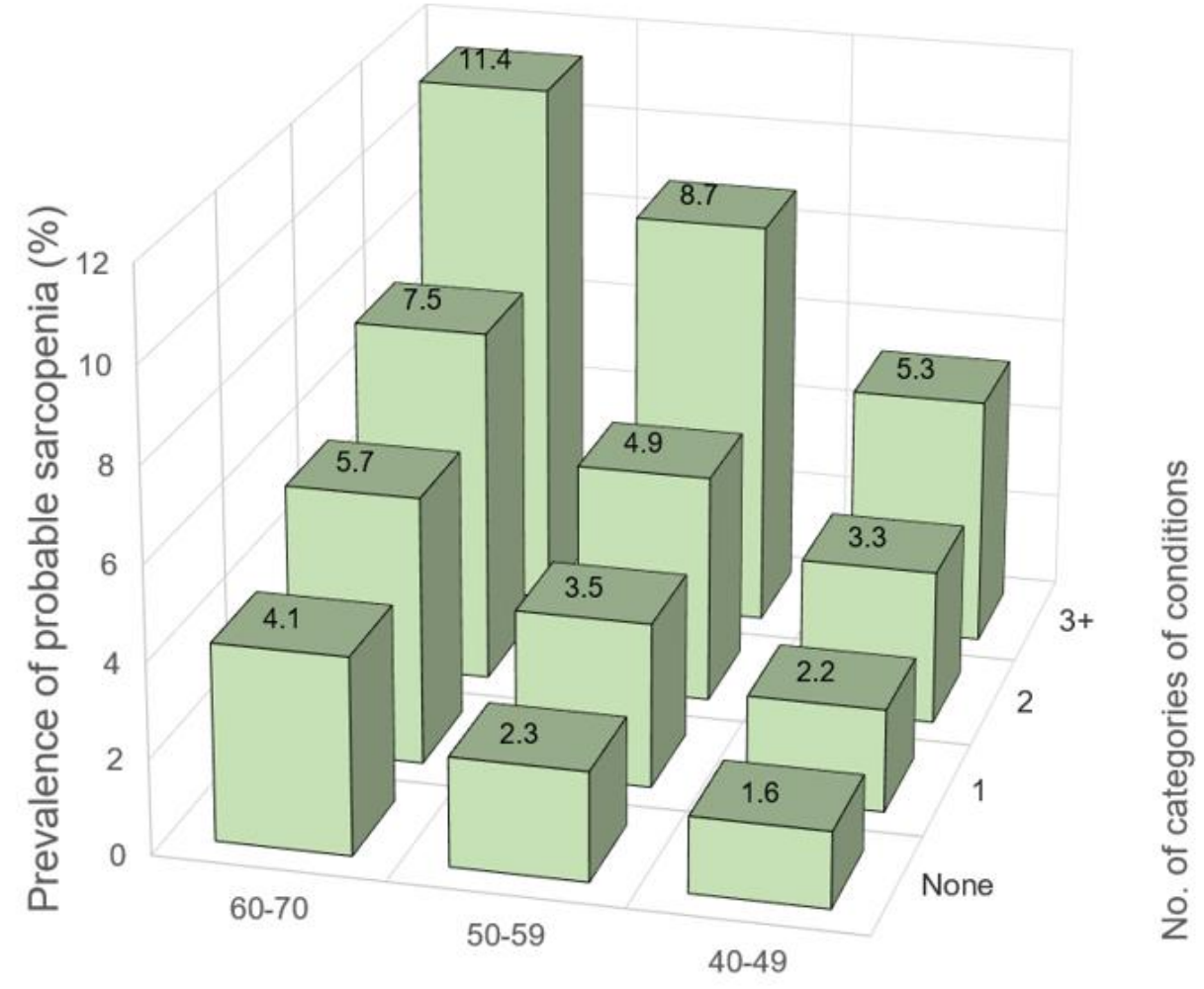

Age group (years)

Findings from UK Biobank. $N=499,046$.

Source: Reproduced from Dodds RM, Granic A, Robinson SM, Sayer AA. Sarcopenia, longterm conditions, and multimorbidity: findings from UK Biobank participants. J Cachexia Sarcopenia Muscle 2020; 11: 62-8. 
Figure 3 Life course normative data for grip strength from 12 British studies.

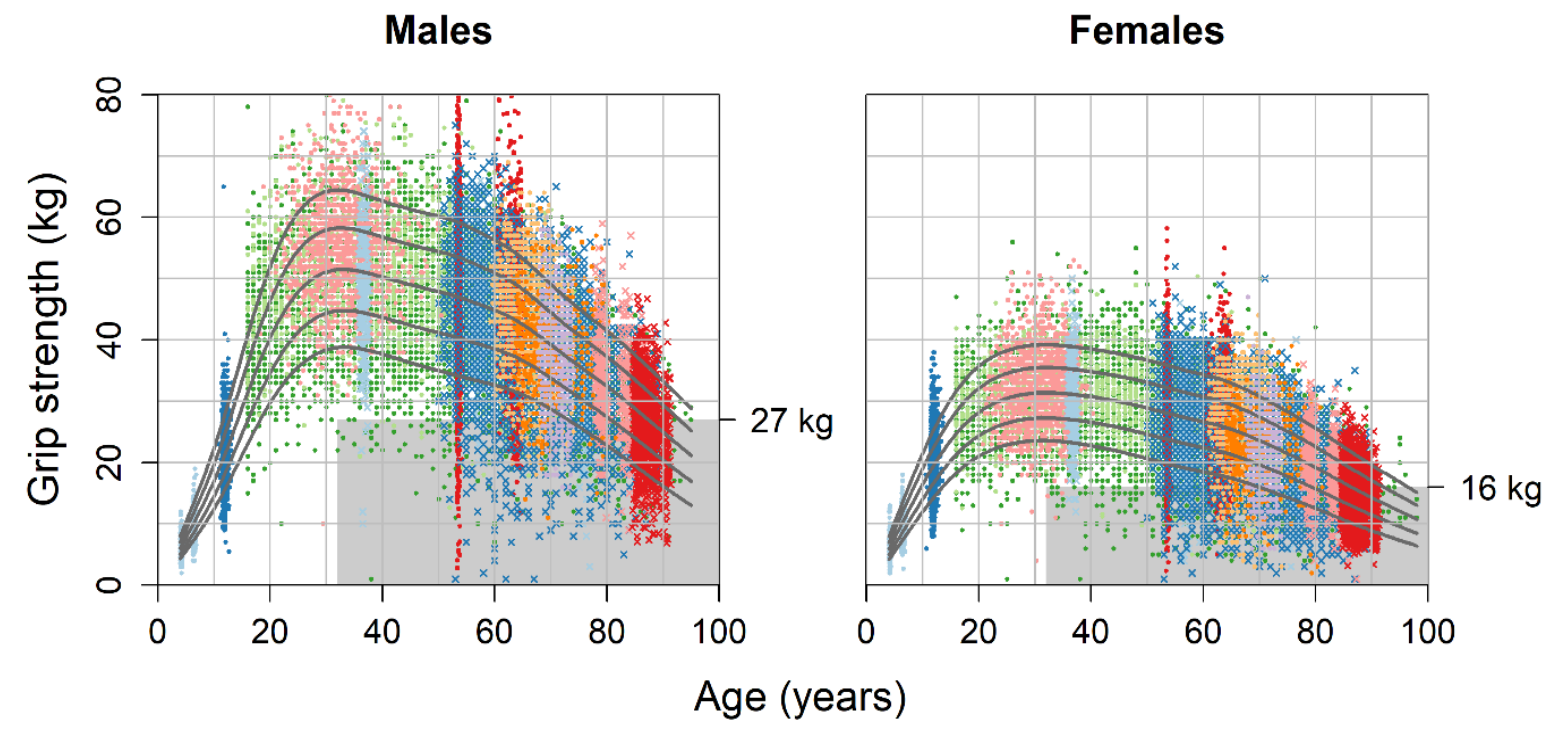

Centiles shown are 10th, 25th, 50th, 75th and 90th. Cut-points based on T-score of $\leq-2.5$ are shown

for male and female participants ( $\leq 27$ and 16kg, respectively). The different markers indicate the British cohort studies used.

Source: Reproduced from Dodds RM, Syddall HE, Cooper R, et al. Grip strength across the life course: normative data from twelve British studies. PLoS One 2014; 9: e113637. 


\title{
TEST YOURSELF
}

To test your knowledge based on the article you have just read, please complete the questions below. The answers can be found at the end of the issue or online here.

\section{Question 1}

An 80-year-old woman presented in the clinic with recurrent falls over the previous 6 months. On clinical assessment, she was noted to struggle rising from a chair, was slow to make her way the short distance to the examination couch and had reduced muscle bulk in the limbs. She screened positively on the SARC-F sarcopenia questionnaire.

\author{
What would be the most appropriate next pair of assessments? \\ A Timed up-and-go test; if time prolonged then arrange an MRI to assess muscle mass \\ B Grip strength with a hand-held dynamometer; if below $16 \mathrm{~kg}$ arrange a DEXA to assess \\ muscle mass \\ C Five chair stand test; if result is more than 10 seconds then check muscle mass using \\ bioelectrical impedance \\ D Grip strength with a hand-held dynamometer; if below $16 \mathrm{~kg}$ then do a short physical \\ performance battery \\ E Assess muscle mass with bioelectrical impedance; if low then test walk speed over $400 \mathrm{~m}$
}

Correct answer: B. The revised European Working Group consensus definition for sarcopenia advises first checking muscle strength, using grip strength or the chair stand test (so $A$ and $E$ are incorrect). The next step is to perform a measurement of muscle mass (so D is incorrect). A slow chair stand test is indicated by taking more than 15 seconds (so $\mathrm{C}$ is incorrect).

\section{Question 2}

A 68-year-old man presented for an annual review with his primary care physician. He had chronic obstructive pulmonary disease, hypertension and chronic kidney disease. The doctor noted that he had carers attending once per day to assist with bathing. The patient was tearful about this, fearing he was losing his independence. He scored 6 - moderately frail - on the Rockwood clinical frailty scale.

\section{What is the best referral route to help him maintain independence?}
A. The respiratory team at his local hospital for assessment of home oxygen
B. His local counselling team for mental health support
C. His local occupational therapy service for equipment at home
D. The local day hospital for holistic, multidisciplinary assessment
E. His local physiotherapy centre for exercise classes

Correct answer: D. The patient has markers of frailty that should trigger arranging for him to have a comprehensive geriatric assessment, with input from all the relevant members of the multidisciplinary team.

\section{Question 3}

A 72-year-old man presented for review shortly after a 2-week hospital admission for biliary sepsis. He had lost $5 \mathrm{~kg}$ in weight, and had found that his exercise tolerance had reduced: he was now only able to walk to the end of his street, but had previously managed the local shops. Going up stairs also took more effort. He remained independent with his activities of daily living and was otherwise well. On examination, the chair stand test was performed slowly, needing 17 seconds to complete five stands.

\section{What is the most appropriate management option to improve his functioning?}
A. Referral to his local physiotherapy centre for resistance exercise classes
B. Prescription of vitamin D supplements
C. Referral to a dietitian for a high-calorie dietary supplements 
D. Prescription of testosterone supplements

E. Prescription of a further course of antibiotics for biliary sepsis

Correct answer: A. Strength and balance exercises are the best way to improve his mobility and independence after his acute illness. There is less evidence of benefit from optimizing nutrition (C) and vitamin $\mathrm{D}(\mathrm{B})$. There is no evidence of benefit from testosterone supplements (D) in this setting. There is no evidence of continuing sepsis (E). 